A N N A L E S Annales de Bretagne et des Pays de l'Ouest

\title{
Envorennou ar barz Juluen Godest. Souvenirs du barde Julien Godest
}

David Hopkin

\section{(2) OpenEdition \\ 1 Journals}

Édition électronique

URL : https://journals.openedition.org/abpo/6959

DOI : 10.4000/abpo.6959

ISSN : $2108-6443$

Éditeur

Presses universitaires de Rennes

\section{Édition imprimée}

Date de publication : 30 juin 2021

Pagination : 218-219

ISBN : 978-2-7535-8359-7

ISSN : 0399-0826

\section{Référence électronique}

David Hopkin, "Envorennou ar barz Juluen Godest. Souvenirs du barde Julien Godest », Annales de Bretagne et des Pays de l'Ouest [En ligne], 128-2 | 2021, mis en ligne le 30 juin 2020, consulté le 15 octobre 2022. URL : http://journals.openedition.org/abpo/6959; DOI : https://doi.org/10.4000/abpo. 6959 
Blanchard, Nelly (dir.), Envorennou ar barz Juluen Godest. Souvenirs du barde Julien Godest, Brest, Éditions du CRBC, 2020, 757 p.

Cette belle édition bilingue d'un manuscrit composé entre 1905 et 1913 permet de découvrir la vie et l'œuvre du paysan et barde breton Julien Godest (1849-1933). On peut faire un parallèle entre ce texte - et la vie de son auteur - et Jean-Marie Déguignet (1834-1905), dont le manuscrit Histoire de ma vie, écrit entre 1898 et 1905, a connu un immense succès lorsqu'il a été publié en 1998 sous le titre Mémoires d'un paysan bas-breton. Les deux hommes ont grandi dans un environnement bretonnant monolingue (Godest dans le nord et Déguignet dans le sud de la Basse-Bretagne) et ont été paysans de condition modeste ; tous deux sont autodidactes dans l'apprentissage de la lecture et de l'écriture non seulement du breton mais aussi du français et dans une certaine mesure du latin, et ce à partir de livres imprimés (notamment ceux de l'imprimeur Lédan à Morlaix). Ils écrivent tous les deux des chansons en breton, et leur entreprise d'écriture autobiographique a été encouragée par la rencontre avec des militants culturels bretons, Anatole Le Braz dans le cas de Déguignet et François Taldir-Jaffrennou pour Godest, qui étaient alors respectivement président et secrétaire de l'Union Régionale Bretonne. C'est d'ailleurs Taldir-Jaffrennou qui a déposé le manuscrit de Godest aux Archives départementales du Finistère. N'ayant jamais été soldat, Godest n'a pas eu l'opportunité de voyager, contrairement à Déguignet, mais son frère Jean-François a été conscrit en 1867 et a servi comme Déguignet en Afrique du Nord puis a participé à la guerre de 1870. L'identité de Godest est profondément imprégnée par ce frère dont le récit des expériences occupe la première partie des Souvenirs.

Il est pourtant peu probable que les Souvenirs de Godest se vendent à plusieurs centaines de milliers d'exemplaires comme les Mémoires de Déguignet, ni qu'ils soient traduits en plusieurs langues, et ce n'est pas parce qu'il a choisi de les écrire en breton. Déguignet est un libre-penseur, radical, esprit fort et anarchiste : ses nombreux actes de rébellion l'ont éloigné de ses contemporains mais l'ont transformé en héros dans sa postérité littéraire. Godest, au contraire, est profondément dévot, adversaire résolu de Combes, des francs-maçons, de l'émigration et de toutes les transformations qui pourraient détourner les paysans bretons de leur loyauté envers l'Église catholique, la langue bretonne et l'ordre social existant dans les campagnes - ces trois éléments étant inextricablement liés dans son esprit. Les Confessions de Rousseau se cachent quelque part dans l'ombre des Mémoires de Déguignet, alors que les modèles littéraires de Godest sont puisés dans la ContreRéforme, comme l'explique Nelly Blanchard dans son excellente introduction. Une bonne partie des Souvenirs est composée de chansons religieuses, elles-mêmes inspirées de cantiques préexistants, même si Godest puise aussi dans d'autres genres établis de longue date dans la littérature religieuse comme le dialogue, l'exemplum, l'interprétation des rêves, l'homélie, le sermon et la prophétie. À l'exception de nombreux remèdes médicinaux recopiés d'après des revues bretonnes, aucun événement ni expérience de sa vie ou de celle de son frère n'échappe à cette dimension morale.

Le résultat peut sembler répétitif, d'autant plus que la morale énoncée est toujours la même : rester chez soi, se contenter de sa condition, honorer Dieu et les saints, parler breton. Par exemple, lorsque son frère conscrit visite en rêve le paradis, il reçoit le don des langues, tel un apôtre, pour raconter et expliquer ce rêve à ses camarades soldats ainsi qu'à un colonel anticlérical et anti-Breton : cette anecdote remplit trente pages du texte. Le projet de Déguignet est de dire les réalités de la vie en Basse-Bretagne en s'en faisant le témoin, là où Godest veut moraliser ce monde. La réalité lui importe peu sauf si elle entretient l'ordre social et divin. 
Il est donc beaucoup plus difficile pour un historien de trouver dans le récit de Godet des informations sur ce que signifie être un enfant qui a faim, un soldat qui se bat pour l'Empire français ou un pèlerin allant au pardon. Et pourtant, il est peut-être un guide plus sûr pour comprendre la mentalité et la culture des paysans bretonnants que Déguignet, qui ne peut cacher son dédain pour les croyances de ses semblables et leur soumission aux hiérarchies sociales et religieuses. Godest, au contraire, partage ces croyances et fait de la soumission une vertu. Parmi les leçons qu'il répète le plus souvent, on trouve l'idée que l'Homme est fait pour travailler et que le paysan est le plus indispensable des travailleurs, ou encore que les pauvres et les humbles sont les intercesseurs privilégiés de Dieu, ceux par qui les miracles arrivent.

Les deux autobiographies proposent chacune une vision bien affirmée et particulière sur la langue bretonne. Déguignet est à la fois obsédé et rebuté par elle, en écho à ce rapport amour-haine qu'il entretient avec lui-même. Il sait qu'elle appartient au plus vaste ensemble des langues celtiques, mais il la décrit régulièrement comme isolée et vouée à l'extinction, délaissée et sans soutien, comme il se présente lui-même. Godest est quant à lui déterminé à préserver cette langue, mais ses affirmations selon laquelle le breton est la langue parlée au jardin d'Éden et au Paradis (ce que met en évidence le rêve de son frère) sont plutôt des réminiscences confuses de théories qui circulaient parmi les celtomanes des XVII ${ }^{\mathrm{e}}$ et XVIII ${ }^{\mathrm{e}}$ siècles. L'importance accordée au breton dans sa pensée peut expliquer pourquoi le clergé est relativement absent de ses écrits, alors même que l'un de ses frères devient prêtre. Pour Godest, un important intercesseur pour faire connaître la volonté de Dieu aux hommes est le barde. Dans un rêve, à la suite de sa rencontre avec TaldirJaffrenou, un joueur de harpe celtique en haut d'une montagne révèle sa vocation à Godest, qui a pour mission de " relever la langue bretonne en Bretagne dans toutes les régions, / Par des cantiques, de belles complaintes, et aussi de jolies chansons ". Il se sent humble et en même temps fier de ce rôle puisqu'il se donne à lui-même le nom de "barde Diamant ".

David HOPKIN

Tumoine, Pascale, Chambet, Denis (dir.), Églises d'llle-et-Vilaine, vingt années de restauration (2000-2019), Rennes, Éditions du département d'Ille-et-Vilaine, 2000, $232 \mathrm{p}$.

Lorsqu'un historien se saisit d'un ouvrage consacré à une politique publique et édité par la collectivité territoriale qui la conduit, il l'ouvre avec circonspection, craignant de voir la communication prendre le pas sur le discours critique. Il a aussi parfois d'heureuses surprises, comme c'est le cas à la lecture de cet ouvrage coordonné par Pascale Tumoine et Denis Chambret, tous deux en charge des questions patrimoniales pour le département d'Ille-et-Vilaine. Entourés d'une équipe de dix-neuf personnes, ils dressent le bilan de la politique patrimoniale du Conseil départemental en faveur des églises au cours des deux dernières décennies.

Les coordinateurs et les concepteurs ont su imaginer un beau livre, tout en couleurs, que l'on survole avec bonheur, à la manière d'un guide touristique richement illustré, en sautant d'un lieu de culte à l'autre. Le lecteur passe ainsi des collégiales aux petites églises rurales, des édifices classés aux monuments non protégés, du gros œuvre aux objets d'art, ce qui le conduit à de belles découvertes. Sur le fond, l'ouvrage se révèle intéressant à plusieurs niveaux. Le principal est qu'il livre une 